\title{
GA Optimization Technique for Portfolio Optimization of Electricity Market Participation
}

\author{
Ricardo Faia, Tiago Pinto, Zita Vale \\ GECAD - Research Group on Intelligent Engineering and Computing for Advanced Innovation and Development \\ Polytechnic of Porto (ISEP/IPP), Porto, Portugal \\ $\{$ rfmfa, tmcfp, zav $\} @$ isep.ipp.pt
}

\begin{abstract}
This paper presents a methodology based on genetic Algorithms (GA) to solve the problem of optimal participation in multiple electricity markets With the emergence of new requirements for electrical power markets, it has become fundamental to develop tools to aid in decision making, understanding the functioning of markets and forecast iterations that occur between the different entities in the market. Artificial intelligence plays a crucial role in the development of these tools. Using artificial intelligence techniques it is possible to simulate the different existing players in the market, to enable these players to be adaptive to any situation, and to model any type of trading. Artificial intelligence based metaheuristic optimization tools allow solving problems in a short time, and with very close results to those that deterministic techniques are able to achieve, at the cost of a high execution time. The achieved results, using a simulation scenario based on real data from the Iberian electricity market, show that the proposed method is able to reach better results than previous implementations of a Particle Swarm Optimization (PSO) and a Simulated Annealing (SA) methods, while achieving very similar objective function results to those of a deterministic approach, in a much faster execution time.
\end{abstract}

Index Terms - Artificial intelligence, Electricity Markets, Genetic Algorithm, Portfolio Optimization

\section{INTRODUCTION}

With the introduction of renewable energy sources in the energy distribution system, the characteristics of this sector have changed completely[1]. The particularities of renewable based generation sources (e.g. intrinsic intermittence and distributed nature, especially in wind and solar generation) not only have an increasing influence on the way the management of the electricity network has to be conducted, but also on the way electricity is commercialized.

With these changes, Electricity Markets (EM) have become more complex and also more competitive. This requires new and effective methods for market operation [1]. In order to deal with the new reality, the concept of Smart Grid (SG) has emerged [2]. SG implementations are growing all around the world [2], [3] considering the management of local generation and consumption independently from the main system, although connected with main grid through a connection bus. With these changes, market regulators and operators are concerned in foreseeing markets' behaviour in order to strengthen market models and operation. Market negotiating

This work is supported by FEDER Funds through COMPETE program and by National Funds through FCT under the projects FCOMP-01-0124-FEDER: PEst-OE/EEI/UI0760/2015; PTDC/SEN-ENR/122174/2010; PTDC/EEAEEL/122988/2010; and SFRH/BD/80632/2011 (Tiago Pinto PhD). players need to understand market rules, with the aim of increasing their profits and reducing their costs [4].

Despite the development in this area, the ability to learn and adapt to provide the best possible results for EM players is still not being properly addressed. In particular, the intelligent use of multiple EM opportunities as they arise is yet a relatively unexplored issue, and should be improved in order to provide players with the capability of optimizing their participation in several simultaneous EM, including the upcoming possibility of negotiating at a SG level.

This paper approaches the portfolio optimization problem for multiple EM participation. The considered model considers real-time adaptation to the most recent events and offers the possibility of buying and selling in the same period, in different EM. With this model, players are able to change their participation profiles at each time. Additionally, real data is used, making the optimization adaptive to the evolution of negotiation contexts throughout the time, by using enhanced market price forecasting methodologies [5]. An evolutionary methodology based on a Genetic Algorithm (GA) [6], [7] is proposed to solve this optimization problem in an acceptable time frame without a significant reduction in the quality of results. The proposed GA approach is also compared with two previous implementation based on the Simulated Annealing (SA) heuristic [8], and Particle Swarm Optimization (PSO) [9], and proves to be able to achieve better objective function results in a similar execution time.

The proposed GA based approach for EM participation portfolio optimization is integrated in AiD-EM (Adaptive Decision Support for Electricity Markets Negotiations) [10], a decision support system for EM market negotiations, which provides the means to test and validate negotiation outcomes in a realistic simulation environment, through the connection to the MASCEM (Multi-Agent Simulator of Competitive Electricity Markets) simulator [11].

After this introductory section, section II presents an overview of the related work in the field, and section III presents the mathematical formulation of the addressed portfolio optimization problem. Section IV details the proposed GA based methodology. Results of a case study based on real data are presented in section $\mathrm{V}$, and the results, including the comparison to previous results are presented in section VI. Finally, section VII presents the most relevant conclusions. 


\section{RELATED WORK}

The optimization of electricity markets participation portfolio represents the allocation of resources (Electricity) in different market opportunities, in a way that the highest possible gain for a negotiating player can be achieved [8]. The traditional approach to the portfolio optimization problem, was initially developed for the activity on the stock exchanges, and only latter has been applied to other areas, such as the electricity markets.

The modern theory management of portfolios "Modern Portfolio Theory" was published in 1952 by Harry Markowitz in an article in Journal of Finance [12] and was subsequently developed in the book Portfolio Selection: Efficient Diversification of investments in 1959. This publication Harry Markowitz secured the award of the Nobel prize in economics sciences in 1990.

The proposed model considers the average of the returns obtained over time, which is obtained by the expected value (average) resulting from the available historical analysis. The expected return of each investment corresponds to the multiplication of the investment to be made by the expected amount. The risk assessment is given by the variance of the portfolio, which measures the variance of the expected return. Adding investments to projects does not only contribute to the expected return, but also interferes with the calculation of the total project risk.

Authors Bar-Lev and Katz [13], were pioneers in the application of the theory of portfolios in power investments. The work entitled "The portfolio approach to Fossil Fuel Procurement in the Electric Utility Industry", analyzes the acquisition of fossil fuels by the US energy industry. With this study, the authors determined how to use energy resources efficiently.

In [14] the portfolio optimization problem is solved by artificial intelligence techniques, namely using GA. In this work, the author presents a portfolio optimization method using different risk measures and in the end makes a comparison between them. All the proposed risk measures derived from the model of the average variance proposed by Markowitz, as follows: a semi-variance model, the mean absolute derivation model and the variance asymmetry. At the end the author present the results through the efficient frontier for each model and for different combinations of assets. The author concludes that GA is a robust and effective method for solving the portfolio optimization problem.

In the work presented in [15], it is demonstrated the applicability of the method mean-variance-skewness (MVS) for portfolios optimization of energy generation company, which is faced with several choices to allocate your energy produced. In this problem the author resorted to techniques of artificial intelligence to solve the problem, using the multiobjective genetic algorithm (MOGAS).

This problem is also extremely relevant when applied to energy markets, especially concerning the support of market negotiating players' decisions. Given the available market opportunities, players need to decide whether to and how participate in each market type, in order to obtain as much gain as possible from their negotiations. This paper thus proposes the optimization of the participation of players in multiple alternative/complementary electricity market types, according to the expected negotiation prices in each moment

\section{MATEMATICAL FORMULATION}

The formulation presented in (1) is used to represent the optimization problem, as proposed in [16] . In (1) $d$ represents the weekday, $N$ day represent the number of days, $p$ represents the negotiation period, Nper represent the number of negotiation periods, Asell $_{M}$ and Abuy $_{S}$ are boolean variables, indicating if this player can enter in negotiation in each market type, $M$ represents the referred market, NumM represents the number of markets, $S$ represents a session of the balancing market, and NumS represents the number of sessions. Variables $p s_{M, d, p}$ and $p s_{S, d, p}$ represent the expected (forecasted) prices of selling and buying electricity in each session of each market type, in each period of each day. The

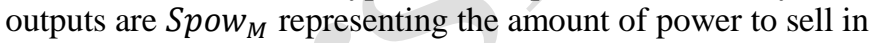
market $M$ and $\mathrm{Bpow}_{S}$ representing the amount of power to buy in session $S$.

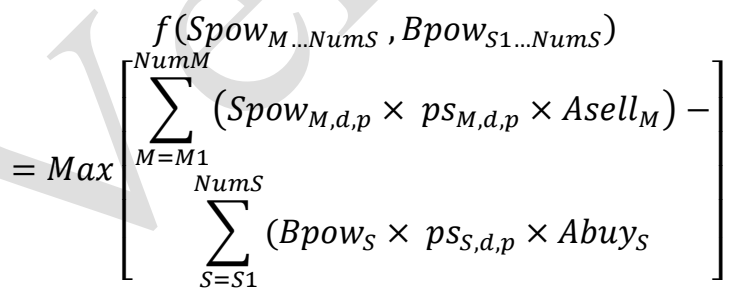

$$
\begin{aligned}
\forall d \in N \text { day, } \forall p & \in \text { Nper }, \text { Asell }_{M} \in\{0,1\}, \text { Abuy } \\
& \in\{0,1\} \\
p s_{M, d, p} & =\operatorname{Value}\left(d, p, \operatorname{Spow}_{M}, M\right) \\
p s_{S, d, p} & =\operatorname{Value}\left(d, p, \text { Bpow }_{S}, S\right)
\end{aligned}
$$

The formulation considers the expected production of a market player for each period of each day. The price value of electricity in some markets depends on the power amount to trade. With the application of a clustering mechanism it is possible to apply a fuzzy approach to estimate the expected prices depending on the negotiated amount. Equation (2) defines this condition.

$$
\begin{array}{r}
\text { Value (day, per, Pow, Market }) \\
=\operatorname{Data}(\text { fuzzy (pow), day,per, Market })
\end{array}
$$

Equation (3) represents the main constraint to be applied in this type of problems, and imposes that the total power that can be sold in the set of all markets is never higher than the total expect production (TEP) of the player, plus the total of purchased power [16]. Further constrains depend on the nature of the problem itself, e.g. type of each market, negotiation amount, type of supported player (renewable based generation, cogeneration, etc.).

$$
\sum_{M=M 1}^{N u m M} \operatorname{Spow}_{M} \leq T E P+\sum_{S=S 1}^{N u m s} \text { Bpow }_{S}
$$




\section{PROPOSED GA APPROACH}

In modern times, businesses are increasingly looking for tools to assist in the negotiation process. Due to the complexity of getting some answers to complex problems in due time, for decision-making, Artificial Intelligence (AI) approaches are used to assist in this process. Within the area of meta-heuristic optimization, Genetic Algorithms (GA) use concepts of evolutionary theory and genetics to search for optimal solutions [17]. The GA is an important tool for optimization solutions, as well as to deal with the demand that involves a large amount of data, especially due to its evolutionary characteristics, which allow performing a combination of answers in order to originate a strong solution.

The GA technique is based on the natural evolution of Darwin, where the most capable individuals survive. The GA's search method tries to find solutions based on probabilities, it does not reach an optimal solution, but looks for a well near solution. Besides the natural evolution, other concepts of biology are applied to the GA, such as the crossover, which is the exchange of information from one individual to another, and mutation where the individual suffers a change in its composition. Natural selection is also used. In Erro! A origem da referência não foi encontrada. it is shown the flow diagram of the GA algorithm [18].

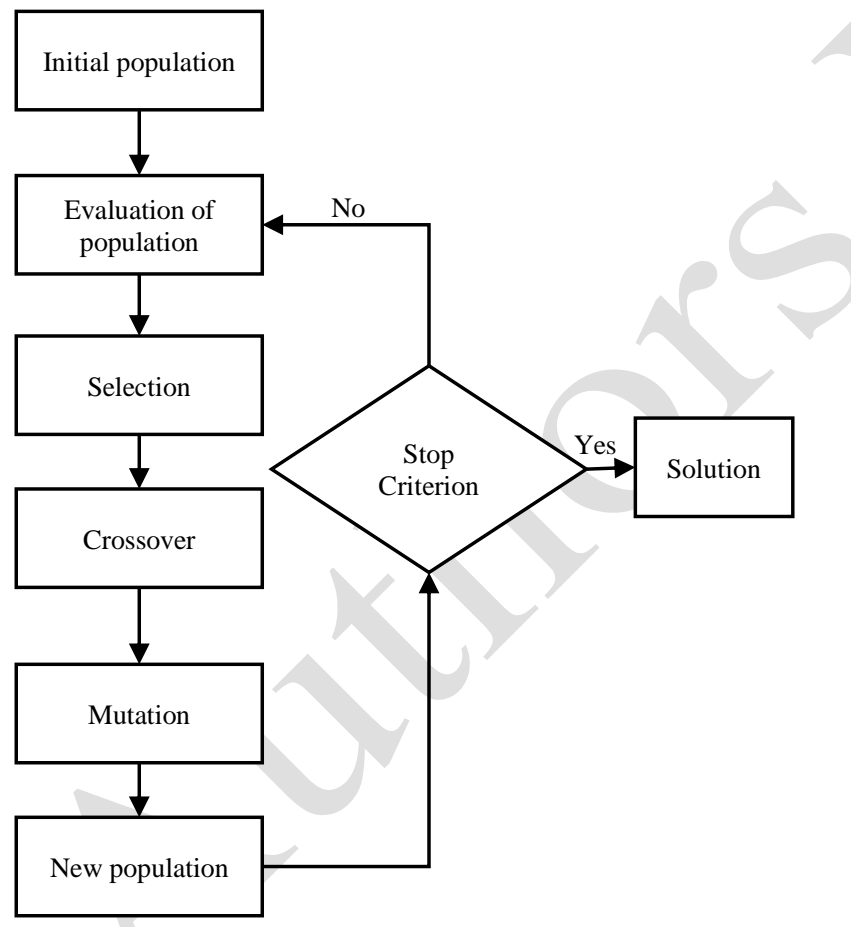

Figure 1. GA flowchart.

The GA process begins with the formation of an initial population, which may be a random population (as is the case in this study). Then, as can be seen in this figure, the population objective functions are assessed, which is done dependently of the problem (in this problem the mathematical formulation presented in section III is used for fitness function evaluation). After the evaluation is made, the individuals are selected; these are the individuals who will be part of the next stage: the crossover process and also the mutation. The new generation of individuals is then found. Following the diagram, follows the stopping criterion, which in this case may be of various natures. If this criterion is satisfied, the algorithm returns the most suitable solution; if this does not happen, the algorithm goes back to the stage of population evaluation, in order to start a new cycle.

In summary, the purpose of the use of GA is to find the individual from the search space with the best "genetic material". The quality of an individual is measured with an evaluation function, called fitness. GA uses a population formed by a set of individuals. Each individual represents a solution or point in the search space. During the evolution, several points are examined by the individuals.

Once the initial population is randomly generated, three operations are used to generate offspring or children to form the following generation [7], as detailed in the following points:

\section{A. Selection}

After evaluation, usually individuals (parents) who will lead to the next generation (children) are selected. The selection follows the concept of natural selection, where the fittest individuals with better playback capabilities. In GA individuals with a better assessment of value will be selected. However, individuals with lower fitness can participate in the selection process to generate children individuals, as well as in nature. The most common methods to make the selection of individuals are the methods of roulette, the tournament and the ranking. In this work, the selection by roulette is applied. The method of roulette [19] favors individuals with better skills, but those with lower fitness can also participate in the process, however with likeliness, thereby always maintaining a random component. The method is implemented in this work as follows:

- The fitness function is evaluated for each individual, providing fitness values, which are then normalized. Normalization means dividing the fitness value of each individual by the sum of all fitness values, so that the sum of all resulting fitness values equals 1 ;

- The population is sorted by descending order of fitness values;

- Accumulated normalized fitness values are computed (the accumulated fitness value of an individual is the sum of its own fitness value plus the fitness values of all the previous individuals). The accumulated fitness of the last individual should be 1 (otherwise something went wrong in the normalization step);

- A random number $R$ between 0 and 1 is chosen;

- The selected individual is the first one whose accumulated normalized value is greater than $R$.

\section{B. Crossover}

After selecting the parent individuals, some individuals will go through the crossover process. This is one of the main methods for the contribution of new individuals. Also called by crossing or recombination, this mechanism is responsible for making the exchange of information between individuals so that new individuals are generated from this recombination 
information. The exchange of information in this work will be performed in two forms, single-point cross and double-point.

Single-point crossover: In this method, a random-cutting point is drawn. Through this cut individuals are divided into two parts. Recombination is made by attaching the first part of the individual with the second part of the second individual.

Two-point crossover: Two points of cuts are randomly drawn. Thus the individual is divided into three parts. The intersection of individuals is made by combining the first and third part of the first individual with the second part of the first individual, and the second part of the second individual with the second part of the first individual, as depicted in Fig. 2. If the drawn point is the starting point or the end, the draw must be done again.

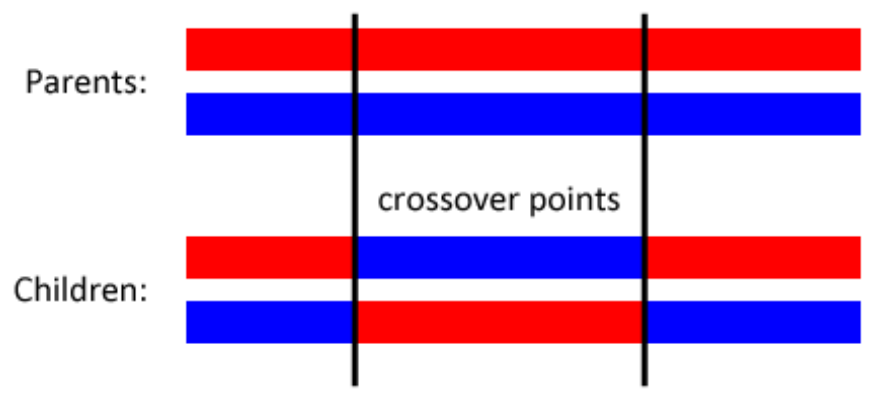

Figure 2. GA's two point crossover.

\section{Mutation}

After the crossover mutation process is conducted, where one or more individuals (who have been selected) are modified. Mutation can be described as a low probability random modification of a chromosome of an individual. The mutation is used to promote genetic diversity over generations. It is analogous to biological mutation observed during meiosis [20] or DNA replication [21]. Mutation may alter one or more genes values in a chromosome (Fig. 3). Hence GA can reach better solutions by using mutation. Mutation occurs during evolution according to a user-definable mutation probability. This probability is normally set to a low value. If it is set too high, the search process will turn into a primitive random search. Nevertheless, this parameter is set by the user in the implemented approach. Another variance that is considered in the present implementation, is: instead of replacing the mutated gene with a random number, it is replaced by the corresponding gene of the individual with the best fitness.

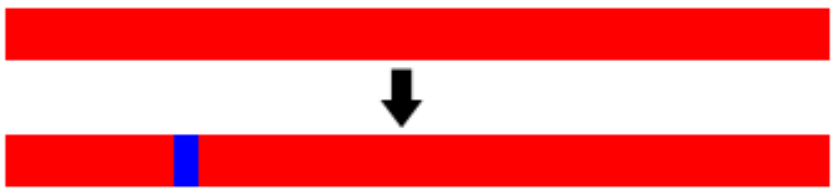

Figure 3. GA's mutation.

After a period of time (number of iterations, also user defined), the most suitable individuals will dominate the population, thus facilitating the achievement of an optimal (or near-optimal) solution.

\section{CAse Study}

This section presents the results of the proposed GA methodology for the resolution of the portfolio optimization problem for multiple complementary/alternative electricity markets participation. The results of the proposed approach are compared to those achieved by a deterministic implementation, which has been modeled using TOMLAB. This problem has been modeled as a mixed integer linear problem (MILP), and has been solved using the cplex solver. The results of the proposed approach are also compared to those achieved by previous implementations using SA [8] and PSO [9].

In order to define a realistic scenario, five different market types have been considered, thereby enabling the supported market player to sell and buy in all of them. The considered markets are the day-ahead spot market, negotiations by means of bilateral contracts, the balancing (or intra-day) market, and a local market, at the Smart-Grid (SG) level. The balancing market is divided into different sessions. In the day-ahead spot market the player (acting as seller) is only allowed to sell electricity, while in the other market types the player can either buy or sell depending on the expected prices. Limits have also been imposed on the possible amount of negotiation in each market. In this case it is only possible to buy up to $10 \mathrm{MW}$ in each market in each period of negotiation, which makes a total of $40 \mathrm{MW}$ purchased. It is possible to sell power on any market, and it can be transacted as a whole or in installments. The player has $10 \mathrm{MW}$ of own production for sale.

In this problem it has also been imposed that in each session of the balancing market, the player can only either sell or buy in each period. In bilateral contracts and in SG negotiation, it is possible to both sell and purchase in the same period (by negotiating with different players).

Since the optimization requires real market data, so that it can be used to support players' decisions in a realistic environment, it is necessary that the electricity prices are provided. The real electricity market prices data, concerning the day-ahead spot market, the intraday market, and bilateral contracts have been extracted from website of the Iberian electricity market operator-MIBEL [22]. The data regarding the considered local market at the SG level refers to previous studies of the authors in this domain [23]. Real data is thereby used by forecasting and estimation methods [24]. In markets where the price of electricity is unique for all participants, regardless of the negotiation amount, an Artificial Neural Network (ANN) is used [25], which is trained with the real historic market prices from MIBEL [R]. In markets where the electricity price is influenced by the negotiated amount (e.g. negotiation by means of bilateral contracts), a prices estimation methodology using fuzzy logic is used [26].

Three versions of the GA based methodology for portfolio optimization have been implemented. The main differences between the three versions are:

- GA 1: uses single-point as crossover approach;

- GA 2: uses the two-point crossover method;

- GA 3: performs mutation according to the maximum fitness. 


\section{RESULTS}

Fig. 4 presents the box plot diagram of the extreme quartiles that represent the results achieved by the three implemented GA versions, after 1000 runs. This type of statistical representation provides an indication of how the data are concentrated. In Fig. 4 the value of the minimum is not shown in order to not hinder comprehension of the higher values.

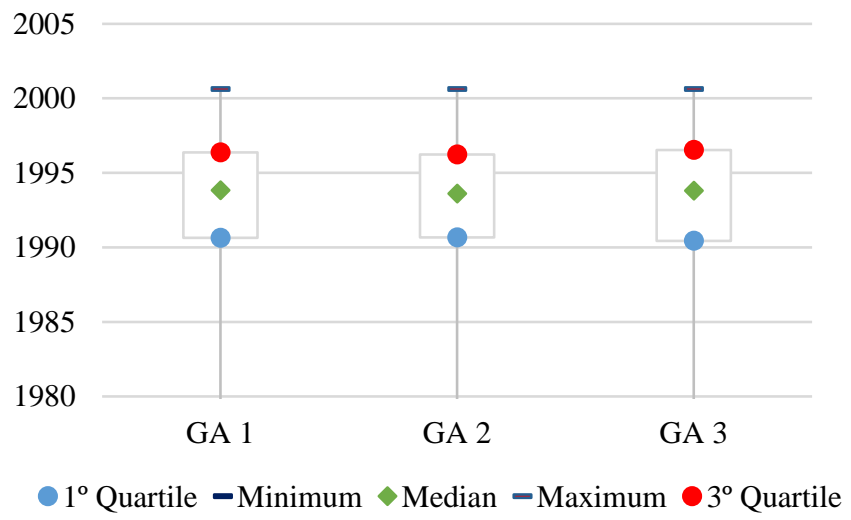

Figure 4. Box plot of the results achieved by the three inplemented GA versions, after 1000 runs.

The distribution of results in these graphs is made as follows: the range from the minimum value to the value of the $1^{\text {st }}$ quartile represents $25 \%$ of the data. Similarly, from the value of the $3^{\text {rd }}$ quartile to the maximum value are also represented another $25 \%$ of the data. Amidst the values of the $1^{\text {st }}$ quartile and $3^{\text {rd }}$ quartile are represented $50 \%$ of the data. From the analysis of the graph it is not easy to draw conclusions about which of the variants presents the best results, as they all present very similar values.

In order to facilitate taking some conclusions on the comparison of performance between the three considered variants, the confidence intervals with a $95 \%$ probability, for each variant, are presented in Figure 5.

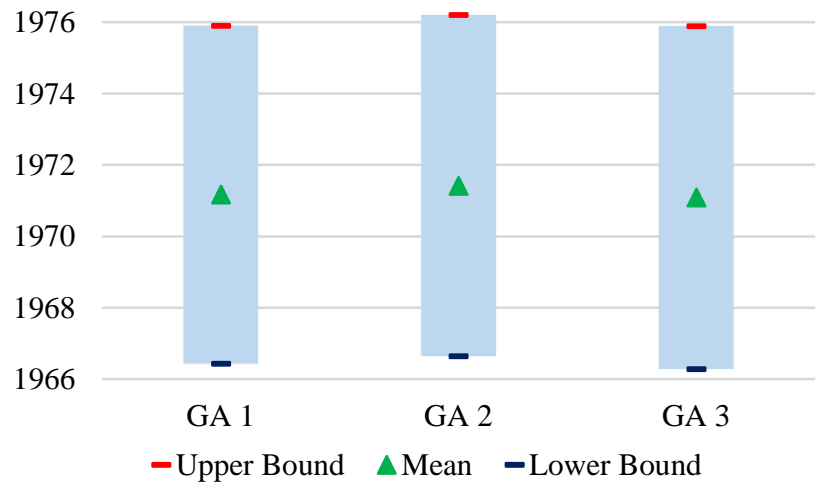

Figure 5. Confidence interval of the results achieved by the three inplemented GA versions, after 1000 runs.

Through the analysis of the confidence intervals, presented in Figure 5, it is possible to see that the GA 2 method has a higher upper and lower limit, as well as a best mean value. Each confidence interval has an associated error; in this case, the GA 2 method has a $4.777 \%$ error. This means that each simulation made with this embodiment has more than $95 \%$ probability of resulting in an objective function value between the upper and lower limits to the determined error. Therefore, the GA 2 approach is chosen as the best GA approach.

Table I shows the scheduling of sales and purchases made in the different markets, as resulting from the best objective function result achieved by the GA 2 variant.

\begin{tabular}{|c|c|c|c|c|c|}
\hline & \multicolumn{5}{|c|}{ GA 2} \\
\hline & Spot & Bila. & Balan. 1 & Balan. 2 & S G \\
\hline $\begin{array}{l}\text { Sales } \\
(\mathrm{MW})\end{array}$ & 14,781 & 11,504 & 0 & 0 & 8,469 \\
\hline $\begin{array}{l}\text { urchases } \\
\text { (MW) }\end{array}$ & 0 & 4,755 & 10 & 10 & 0 \\
\hline
\end{tabular}

Fig. 6 shows the price variation by trading volume in period 1. As can be seen, some markets (spot and balancing) present a unique market price for this hour, while bilateral and SG present prices that are variable with the negotiated amount. The expected result is that the optimization allocates the sales to markets and amounts where the price is higher and purchases when the price is lower.

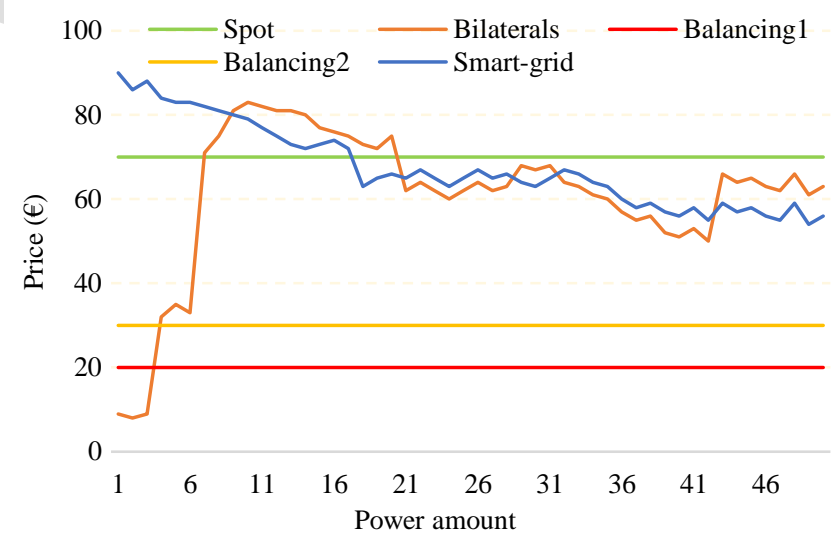

Figure 6. Price variation in the considered markets for the first period of the day, depending on the negotiated amount of power

By matching Table I and Fig. 6 it is possible to explain the functioning the operation of this methodology. As expected, the model presents a solution with the purchased energy in the cheapest markets and sales in the most profitable. As the total energy that can be bought in each market is $10 \mathrm{MW}$, the maximum amount is bought in the balancing sessions (lower prices), and also a purchase of $4.755 \mathrm{MW}$ in bilateral contracts. The sale is set to the SG in $8.469 \mathrm{MW}, 11.504 \mathrm{MW}$ in bilateral contracts and 14.781 MW in the spot market.

Table II presents a comparison of the objective function results achieved in the total of 1000 runs, by the GA approaches against the results from the previous SA, PSO and deterministic implementations. Table III presents the comparison of the average execution time achieved by the different methods. 
Table II - Comparison between the GA approach and the other algorithms objective function results

\begin{tabular}{|c|cccc|}
\hline \multirow{2}{*}{ Algorithm } & \multicolumn{4}{|c|}{ Objective value $(€)$} \\
\cline { 2 - 5 } & Min. & Mean & Max. & STD \\
\hline Deterministic & & & 2000,65 \\
PSO & 935,04514 & 1802,21 & 2000,634 & 160,42349 \\
SA & 1781,4805 & 1884,04 & 1927,2421 & 55,50148 \\
GA 1 & 1545,4513 & 1971,173 & 2000,624 & 76,409591 \\
GA 2 & 1453,132 & 1971,4222 & 2000,6147 & 77,121964 \\
GA 3 & 1444,3912 & 1971,0881 & 2000,622 & 77,534255 \\
\hline
\end{tabular}

Table III - Comparison of execution times of the GA approach, deterministic method, and previous SA and PSO methods

\begin{tabular}{|c|c|}
\hline Algorithm & Time (seconds) \\
\hline Deterministic & 41040 \\
PSO & 1,0246353 \\
SA & 0,5191096 \\
GA 1 & 5,0939918 \\
GA 2 & 5,9576606 \\
GA 3 & 5,6625342 \\
\hline
\end{tabular}

How it is possible to observe by Table II, the results achieved by all three proposed GA based methodologies feature significant advantages when compared to the other approaches. The minimum results achieved by the three alternative GA methods are much higher than the results of the PSO metaheuristic technique. The GA algorithms are also the ones that are able to achieve the best mean values from all algorithms. The maximum achieved value, is however, just a little below the result achieved by the PSO and by the deterministic approach. Nevertheless, the values achieved by GA are very close to these objective function results. Consequently, the Standard Deviation (STD) assumes very low values when compared to the PSO approach. Therefore, the proposed GA based methods are a more reliable alternative than the PSO, and able to achieve better results than the SA based approach.

In what concerns the execution time, as it can be seen by Table III, GA needs more time to reach a solution than the other two metaheuristic algorithms, but it is still incomparably faster than the deterministic approach. In fact, the execution time of the deterministic approach is higher than acceptable, as it refers to several hours of simulation, which cannot be usable for decision support of players' negotiations, when negotiations occur in much smaller time constraints. The proposed GA approach takes only nearly 5 seconds to reach a near-optimal solution (for each period of negotiation), which is highly acceptable for the case in matter.

\section{CONCLUSIONS}

The resolution of the portfolio optimization problem in a multiple electricity markets environment is essential for electricity market negotiation players, so that they can be able to take as much benefit from market negotiations as possible. Addressing this problem is particularly relevant considering the constant rate at which market rules and mechanisms are changing, especially with the large penetration of distributed generation.

This paper has proposed a GA based approach to solve this optimization problem in an acceptable time frame without a significant reduction in the quality of results. The proposed GA approach has been compared with previous implementations based on PSO and SA, and it has shown to be more reliable than the previous methods, and guarantee a higher mean objective function value. The maximum achieved value is very close to the deterministic method results. Despite the AG take longer than the PSO and SA, this is nevertheless invalid because each period is one hour and the AG runtime never overcomes. The execution time required to achieve such results are still much below the execution time of the deterministic approach, and thereby the proposed method proves to be adequate for its decision support purposes.

As future work, the creation of the initial population will be explored, namely by implementing an heuristic method to return an initial solution based on trading rules of the markets, in order to try and enhance even further the results and execution time of the proposed method.

\section{REFERENCES}

F. P. Sioshansi, Evolution of Global Electricity Markets: New Paradigms, New Challenges, New Approaches. 2013.

H. Lund, A. N. Andersen, P. A. Østergaard, B. V. Mathiesen, and D. Connolly, "From electricity smart grids to smart energy systems - A market operation based approach and understanding," Energy, vol. 42, no. 1, pp. 96-102, 2012.

[3] T. Pinto, H. Morais, Z. Vale, and I. Praça, "Multi-Agent Negotiation for Coalition Formation and Management in Electricity Markets," in Negotiation and Argumentation in Multi-Agent Systems Fundamentals, Theories, Systems and Applications, H. C. F. Lopes, Ed. Bentham e-books, 2014, pp. 334-360.

[4] T. Pinto, Z. Vale, F. Rodrigues, I. Praça, and H. Morais, "Multiagent system for adaptive strategy formulation in electricity markets," Intelligent Agent (IA), 2011 IEEE Symposium on. pp. 1-8, 2011.

[5] T. Pinto, T. M. Sousa, I. Praça, Z. Vale, and H. Morais, "Support Vector Machines for decision support in electricity markets' strategic bidding," Neurocomputing, vol. 172, pp. 438-445, Jan. 2016.

[6] I. Lee, R. Sikora, and M. J. Shaw, "A genetic algorithm-based approach to flexible flow-line scheduling with variable lot sizes," IEEE Transactions on Systems, Man, and Cybernetics, Part B (Cybernetics), vol. 27, no. 1. pp. 36-54, 1997.

[7] D. E. Goldberg, Genetic Algorithms in Search, Optimization and Machine Learning, 1st ed. Boston, MA, USA: Addison-Wesley Longman Publishing Co., Inc., 1989. 
R. Faia, T. Pinto, and Z. Vale, "Optimization of Electricity Markets Participation with Simulated Annealing," in Trends in Practical Applications of Scalable Multi-Agent Systems, the PAAMS Collection, F. de la Prieta, J. M. Escalona, R. Corchuelo, P. Mathieu, Z. Vale, T. A. Campbell, S. Rossi, E. Adam, D. M. Jiménez-López, M. E. Navarro, and N. M. Moreno, Eds. Cham: Springer International Publishing, 2016, pp. 27-39.

[9] T. Pinto, H. Morais, T. M. Sousa, T. Sousa, Z. Vale, I. Praça, R. Faia, and E. J. S. Pires, "Adaptive Portfolio Optimization for Multiple Electricity Markets Participation," Neural Networks Learn. Syst. IEEE Trans., vol. PP, no. 99, p. 1, 2015.

[10] T. Pinto, Z. Vale, F. Rodrigues, H. Morais, and I. Praça, "Bid Definition Method for Electricity Markets Based on an Adaptive Multiagent System," in Advances on Practical Applications of Agents and Multiagent Systems: 9th International Conference on Practical Applications of Agents and Multiagent Systems, Y. Demazeau, M. Pěchoucěk, J. M. Corchado, and J. B. Pérez, Eds. Berlin, Heidelberg: Springer Berlin Heidelberg, 2011, pp. 309-316.

[11] G. Santos, T. Pinto, I. Praça, and Z. Vale, "MASCEM: Optimizing the performance of a multi-agent system," Energy, vol. 111, pp. 513-524, 2016.

[12] H. Markowitz, "PORTFOLIO SELECTION*," J. Finance, vol. 7, no. 1, pp. 77-91, Mar. 1952.

[13] D. Bar-Lev and S. Katz, "A Portfolio Approach to Fossil Fuel Procurement in the Electric Utility Industry," J. Finance, no. American Finance Association, pp. 933-47, 1976.

[14] T.-J. Chang, S.-C. Yang, and K.-J. Chang, "Portfolio optimization problems in different risk measures using genetic algorithm," Expert Syst. Appl., vol. 36, no. 7, pp. 10529-10537, Sep. 2009.

[15] K. Suksonghong, K. Boonlong, and K.-L. Goh, "Multi-objective genetic algorithms for solving portfolio optimization problems in the electricity market," Int. J. Electr. Power Energy Syst., vol. 58, pp. 150-159, 2014.

[16] T. Pinto, Z. Vale, T. M. Sousa, T. Sousa, H. Morais, and I. Praça, "Particle Swarm Optimization of Electricity Market Negotiating Players Portfolio," Highlights Pract. Appl. Heterog. Multi-Agent Syst., vol. 430, pp. 273-284, 2014.
[17] V. L. Policicchio, A. Pietramala, and P. Rullo, "GAMoN: Discovering M-of- hypotheses for text classification by a latticebased Genetic Algorithm," Artif. Intell., vol. 191-192, pp. 61-95, Nov. 2012.

[18] J. Holland, "Genetic algorithms," Sci. Am., pp. 66 - 72, 1992.

[19] L. M. Schmitt, "Theory of Genetic Algorithms II: models for genetic operators over the string-tensor representation of populations and convergence to global optima for arbitrary fitness function under scaling," Theor. Comput. Sci., vol. 310, no. 1-3, pp. 181-231, Jan. 2004.

[20] H. Cederberg and U. Rannug, "Mechanisms of human minisatellite mutation in yeast," Mutat. Res. Mol. Mech. Mutagen., vol. 598, no. 1, pp. 132-143, 2006.

[21] J. S. Bertram, "The molecular biology of cancer," Mol. Aspects Med., vol. 21, no. 6, pp. 167-223, 2000.

[22] MIBEL, "Mercado Iberico de Eletrecidade," 27-Feb-2007. [Online]. Available: http://www.mibel.com/index.php?lang=pt. [Accessed: 27-Feb-2016].

[23] H. Morais, T. Pinto, Z. Vale, and I. Praça, "Multilevel Negotiation in Smart Grids for VPP Management of Distributed Resources," Intelligent Systems, IEEE, vol. 27, no. 6. pp. 8-16, 2012.

R. Faia, T. Pinto, and Z. Vale, "Dynamic Fuzzy Clustering Method for Decision Support in Electricity Markets Negotiation," Adv. Distrib. Comput. Artif. Intell. J., vol. 5, no. 2, 2016.

[25] T. Pinto, T. M. Sousa, and Z. Vale, "Dynamic artificial neural network for electricity market prices forecast," Intelligent Engineering Systems (INES), 2012 IEEE 16th International Conference on. pp. 311-316, 2012.

[26] R. Faia, T. Pinto, and Z. Vale, "Dynamic Fuzzy Estimation of Contracts Historic Information Using an Automatic Clustering Methodology," in Highlights of Practical Applications of Agents, Multi-Agent Systems, and Sustainability - The PAAMS Collection SE - 23, vol. 524, J. Bajo, K. Hallenborg, P. Pawlewski, V. Botti, N. Sánchez-Pi, N. D. Duque Méndez, F. Lopes, and V. Julian, Eds. Springer International Publishing, 2015, pp. 270-282. 